\title{
Anna Siyanova-Chanturia On the 'holistic' nature of formulaic language
}

\begin{abstract}
Many applied and corpus linguists entertain the idea of collocations, and other types of formulaic language, being processed as unanalysed, or holistic units. It has, indeed, been demonstrated that, due to their frequency and predictability, formulaic sequences are processed quantitatively faster than matched novel phrases. This finding implies an important role of phrasal frequency in language processing and highlights the contribution of entrenchment of a particular phrasal configuration in memory. This finding, however, cannot be taken to suggest that formulaic sequences are necessarily processed as unanalysed, or holistic units. The present paper reviews some of the recent studies and explains why a processing advantage observed for formulaic sequences over novel phrases should not be equated with holistic storage and processing. ${ }^{1}$
\end{abstract}

Keywords: formulaic language, frequency, holistic storage, on-line processing, usage-based theories

DOI 10.1515/cllt-2014-0016

Recent years have seen a growing interest in the research that focuses on various kinds of formulaic language, ${ }^{2}$ their representation and on-line processing. ${ }^{3}$

1 The present paper is not intended as an overview of the studies on on-line processing of formulaic language. For a comprehensive review of the method and findings specific to formulaic sequences, their on-line representation and processing, we direct an interested reader to Siyanova-Chanturia (2013) and Siyanova-Chanturia and Martinez (2014).

2 In the present paper, we will use the terms formulaic sequences and formulaic language interchangeably. When discussing specific studies, various types of formulaic language will be referred to by their own names (e.g. idioms, collocations, phrasal verbs, lexical bundles, etc.).

3 We define on-line processing as processing happening in real time. In on-line studies, reaction times and/or brain activity are recorded while participants perform a task under significant time pressure.

Anna Siyanova-Chanturia, School of Linguistics and Applied Language Studies, Victoria University of Wellington, Wellington, New Zealand, E-mail: anna.siyanova@vuw.ac.nz 
Formulaic language can be of many different kinds, such as, collocations (fast food), binomials (black and white), multi-word verbs (rely on), idioms (tie the knot), speech formulae (what's up?), discourse markers (by the way), lexical bundles (as well as), expletives (damn it!), grammatical constructions (the -er the -er), and many more. Formulaic language is important because it constitutes a large proportion of authentic native-speaker discourse. According to some estimates, between $20 \%$ and $50 \%$ of language we encounter on a daily basis is formulaic (e.g. Biber et al. 1999; Erman and Warren 2000).

One of the most often cited and adopted definitions of a formulaic sequence is that of Wray (2002), according to whom, it is:

... a sequence, continuous or discontinuous, of words or other elements, which is, or appears to be, prefabricated: that is, stored and retrieved whole from memory at the time of use, rather than being subject to generation or analysis by the language grammar. (Wray 2002: 9)

As Wray (2002) points out, this definition aims to be as inclusive as possible, covering a wide range of linguistic units that have been or can be considered formulaic. In her discussion of the lexicon, Wray (2002: 266) seems to go a step further proposing that units of different sizes and internal structure can be stored in the lexicon as "morpheme equivalents". This proposition is further developed in Wray (2008), where she puts forward the concept of a morpheme equivalent unit, which is defined as:

a word or word string, whether incomplete or including gaps for inserted variable items, that is processed like a morpheme, that is, without recourse to any form-meaning matching of any sub-parts it may have. (Wray 2008: 12)

As Wray (2008: 113) notes, "the definition of the morpheme equivalent unit [...] captures what is believed to be happening in language processing" (emphasis added). Correspondingly, the discussion below will be centred around the issues pertinent to the processing of formulaic language.

The key tenets of the above, not dissimilar, definitions are that instances of formulaic language are, or appear to be, "stored and retrieved whole from memory, [...], rather than being subject to generation or analysis by the language grammar" (Wray 2002: 9) and that they behave akin to a single morpheme (Wray 2008). Wray (2002, 2008) is not alone in this view. Researchers, long before but particularly after Wray (2002), have claimed that various types of formulaic language are stored and processed holistically, although they have not always been explicit about their description of holistic storage and processing (e.g. Altenberg 1998; Dahlmann and Adolphs 2007; Pawley and Syder 1983; Raupach 1984; Schmitt and Carter 2004; Spöttl and McCarthy 2004). 
With the two definitions in mind, let us, first, consider what being "stored and retrieved whole from memory" (Wray 2002: 9) and being "processed like a morpheme" (Wray 2008: 12) means and what implications these propositions may have for on-line language processing. Such definitions of holistic storage, retrieval, and processing, and morpheme-like status entail that formulaic sequences are processed (comprehended and produced) as a unitary whole, that is, without access to or analysis into their constituents. In other words, holistic storage presupposes that a formulaic sequence is represented and processed independently of the properties (lexical, semantic, syntactic, etc.) of its constituent parts. Put simply, these definitions imply that when encountering a highly recurrent phrase, such as, for example, fast food, we do not necessarily activate, or access in our mental lexicon, the individual components of this phrase - fast and food; rather, we process the chunk fast food as one holistic, morpheme-like unit. ${ }^{4}$ To investigate whether or not the individual words are activated, or play a role in the processing of the whole, or if the phrase is amendable semantically or syntactically, one would need to employ, for example, a semantic or syntactic priming paradigm, or explore the effect of individual word frequency relative to the frequency of the formulaic sequence.

Let us now consider the evidence for holistic storage that some studies report to have found. In an eye-tracking study, Underwood et al. (2004) found that the terminal words in idiomatic expressions were read more quickly than the same words when in non-idiomatic contexts. Based on this finding, the authors concluded that idioms were stored and processed holistically:

We now have evidence that the terminal words in formulaic sequences are processed more quickly than the same words when in nonformulaic contexts. This provides evidence for the position that formulaic sequences are stored and processed holistically. (Underwood et al. 2004: 167)

In another study, Jiang and Nekrasova (2007) compared grammaticality judgments for frequent phrases against those of control phrases, and found the former to be both faster and more accurate than the latter. Based on this result, the authors concluded that formulaic sequences were recognised holistically and were not subject to the full syntactic analysis (which, it was argued, necessarily takes place for novel phrases):

4 Wray (2002) acknowledges that a formula may be broken down and analysed into its constituent parts. However, this only happens when needs require it; that is, it is not a default procedure. A default, according to Wray (2002), is when a formulaic sequence is not analysed or segmented into its individual components and, as a result, it is stored and retrieved holistically. 
... the NSs and the NNSs of English responded to formulaic expressions significantly faster than they did to nonformulaic expressions [...] Our findings provide clear and straightforward support for a holistic view of formula representation and processing. (Jiang and Nekrasova 2007: 441-2)

In a more recent self-paced reading study, Kim and Kim (2012) investigated the effect of phrasal frequency on the processing of two-word verbs (e.g. find out, sort out). Similar to the above studies, it was concluded that frequent multi-word verbs were stored as unanalysed units - again, solely on the basis of having found longer reading times for the lower frequency items.

Although many applied and corpus linguists agree that formulaic language is "fundamentally a psychological concept" (Hoey 2005: 7), and that the idea that formulaic sequences are stored holistically in the mental lexicon is a "psycholinguistic claim" (Millar 2011: 130), "advanced in the area of psycholinguistics" (Dahlmann and Adolphs 2007: 49), unfortunately, applied and corpus linguists themselves do not always draw on the research that exists in the field of psycholinguistics. Let us take, for example, the recent study by Kim and Kim (2012) mentioned above. This paper cites only one study on formulaic language processing published in a psychology journal - Sosa and MacFarlane (2002) - which is, admittedly, one of the very first studies on this topic. None of the recent studies that appeared in psychology journals (e.g. Arnon and Snider 2010; Bannard and Matthews 2008; Molinaro and Carreiras 2010; Vespignani et al. 2010) made it to Kim and Kim (2012). Neither are any of these studies - not even the early study by Sosa and MacFarlane (2002) - cited in another recent paper by Millar (2011), who, nevertheless, claims to have adopted a "psycholinguistic perspective" (Millar 2011: 129-30). A quick look at another recent paper by Edmonds (2014) suggests that it is too thin on psycholinguistic evidence. Although on-line processing of the various types of formulaic language (in particular, those that are not idioms; idioms have long been of interest to linguists, psycholinguists, and neurolinguists alike) has recently become a popular topic of investigation in applied and psycholinguistic research, there are still relatively few studies on the topic. Due to the relative novelty and scarcity of such studies, and precisely because formulaic language is "fundamentally a psychological concept" (Hoey 2005: 7), it is imperative for researchers to be familiar with and draw on the existing body of evidence not only in their own field, but also in the neighbouring disciplines (such as, for example, psycholinguistics and neurolinguistics).

Another issue pertains to the fact that some researchers believe that evidence for the holistic storage of formulaic language can come from (written) corpus studies alone (e.g. Herbst 2011). However, as Durrant (2008: 132) points 
out, "the link from corpus to mind is likely to be at best an indirect one", and as Durrant and Siyanova-Chanturia (in press) further emphasise:

\begin{abstract}
Corpus-only studies which attempt to draw psycholinguistic conclusions rely on theoretical assumptions which are often open to debate and so should be treated as correspondingly tentative. It is especially important that we avoid the temptation of automatically ascribing all patterns found in the corpus to features of the mind without further interrogation. (Durrant and Siyanova-Chanturia, in press)
\end{abstract}

It then follows that researchers have argued in favour of holistic storage either on the basis of their theoretical assumptions, or on the grounds of having found a significant processing advantage for a particular kind of formulaic language over matched control phrases. However, it is important to bear in mind that the processing advantage per se cannot shed much light on the holistic storage of formulaic language (or lack thereof). The studies that claim to have found evidence for holistic storage (e.g. Jiang and Nekrasova 2007; Kim and Kim 2012; Millar 2011; Underwood et al. 2004) were simply not designed to explore the holistic status issue, not least because none of these studies investigated the activation of the individual components within a formulaic sequence. Rather, the designs employed addressed the question of the speed of processing of formulaic sequences versus matched control phrases.

What such a processing advantage does show is that formulaic sequences are processed (i.e. comprehended and produced) quantitatively faster than control phrases, due to being frequent and, thus, highly familiar and predictable strings of language. This processing advantage has been taken to suggest that language users notice, learn, and store frequency information not only about single words but also about phrases (e.g. Arnon and Snider 2010; SiyanovaChanturia et al. 2011b; Snider and Arnon 2012), and that each and every occurrence of a linguistic form, at the word or phrase level, contributes to its degree of entrenchment in a speaker's memory (e.g. Bybee 1998, Bybee 2006; SiyanovaChanturia et al. 2011b). As such, this finding has been taken to support usagebased and exemplar-based approaches to language acquisition, processing, and use (Bod 1998, Bod, 2006; Bybee 1998; Goldberg 1995, Goldberg 2006; Langacker 1987; Pierrehumbert 2001; Tomasello 2003), according to which the allocation of representations to linguistic exemplars is accomplished on the basis of frequency, and, thus, language should be viewed not as a set of specific grammar rules, but as a statistical accumulation of linguistic experiences (Bod 2006). So, what phrasal frequency effects - both in production and comprehension reflect is hundreds and thousands of previous encounters with a given phrase. Because our experiences with linguistic events are constantly changing, the way in which we process and represent these events changes too, as has been shown 
in the studies with second language (L2) learners (Siyanova-Chanturia et al. 2011b; Siyanova-Chanturia and Spina in press). For example, SiyanovaChanturia et al. (2011b) found that the more proficient L2 speakers (i.e. those with more experience with the L2) were more sensitive to the frequency information encoded in the target phrases and their processing was, thus, more akin to that of native speakers, compared to the less proficient L2 speakers (i.e. those with less experience with the L2).

All in all, a processing advantage observed for formulaic sequences over novel phrases in reading and production studies is a vital piece of evidence which argues for the crucial role of phrasal frequency in language acquisition, processing, and use and which has shed important light on the nature of the mental lexicon; but which cannot, on its own, warrant conclusions favouring the holistic storage debate, not least because this finding tells us nothing about the behaviour - activation and prominence - of the individual components within a phrase.

It was noted above that holistic storage and processing implies that a formulaic sequence is processed as one single, morpheme-like unit; that is, without access to, or analysis into, its constituent parts. The question of whether or not individual parts are activated within a larger unit, or play a role in its processing, has been addressed in the research on compounds. Compounds are, in many ways, akin to formulaic sequences, in that a larger unit is comprised of smaller meaningful ones (e.g. bedroom, bluebird, blackboard). In addition, some compounds are spelled as two words (e.g. red cross, apple pie, life style), rendering them similar to some formulaic sequences (e.g. collocations). ${ }^{5}$ The critical question in compound research has been whether or not such strings are decomposed into their constituents during on-line processing (e.g. bed + room; blue + bird; black + board). According to Badecker (2001), the processing of compounds takes a purely compositional approach. Juhasz (2007) and Libben (1998) also argue that compound structures are decomposed during their recognition. Taking a slightly different approach, Pollatsek et al. (2000) maintain that the representation of a compound can be accessed via its individual lexemes (e.g. bed and room) as well as via the entire compound (e.g. bedroom). Similarly, Mondini et al. (2005) propose that lexical retrieval of a compound entails the activation of its individual components and the whole form of the compound (also see Kuperman et al. 2008, Kuperman et al. 2009). Overall, the research on compounds agrees that individual constituents can be activated and accessed in the mental lexicon during compound processing, irrespective of whether a

5 It needs to be noted, however, that compounds and formulaic sequences may differ in the degree of compositionality. 
purely compositional or a parallel access approach is adopted. Unfortunately, despite certain similarities between compounds and some instances of formulaic sequences (e.g. collocations), very few, if any, studies on formulaic language online processing review, or make any reference to, the wealth of psycholinguistic research on compound processing.

Although the majority of the studies that have looked at on-line processing of formulaic language (in particular, those that claim to have found evidence for holistic storage) have found solely on the speed of processing and have not examined the activation or prominence of the individual components within a larger chunk, there are, nevertheless, three studies that can be said to be an exception, in that they have directly considered the relationship between the parts and the whole. In the earliest such study, Sosa and MacFarlane (2002) monitored the response to the particle of in two-word phrases that varied in frequency (e.g. kind of, because of, sort of). These authors found that reaction times to the particle of in higher frequency phrases were slower and the accuracy was lower than in lower frequency phrases. Sosa and MacFarlane (2002) concluded that frequent phrases were treated as a unit, and hence the access to one of its constituents (i.e. of) was impeded. However, the study suffers from a number of limitations. Although the particle of was kept constant in all target phrases - higher and lower frequency ones - the lexical properties of the constituent words within the target phrases were not controlled for. Critically, the experimental items, extracted from the Switchboard corpus, were not controlled for the duration of the particle of. Thus, detection was likely to have been hindered simply because the phonetic duration of the particle of was shorter in higher frequency phrases (where the consonant may not be pronounced at all and, as a result, the particle may appear acoustically non-salient) than in lower frequency ones. In another study employing a similar paradigm, Kapatsinski and Radicke (2009) had participants detect the particle up in verb + up phrases varying in frequency (e.g. sign up, give up, hold up), where the phonetic duration of the particle was controlled for. The authors observed a U-shaped pattern: detection of the particle up was slower when the frequency of the verb + up phrase was either very low or extremely high compared to when it was in the middle of the frequency continuum. Although more carefully conducted than the study by Sosa and MacFarlane (2002), this study has too been criticised in the literature. For example, Arnon and Cohen Priva (in press) point out that because only one particle was looked at, the findings reported cannot shed light on the relationship between individual word and phrase frequency, and because only two-word phrases were investigated, it is possible that what the findings reflect is, in fact, word-to-word transitional probabilities rather than multi-word information. 
Despite these limitations, the findings of Sosa and MacFarlane's (2002) and Kapatsinski and Radicke's (2009) studies are important in that they imply that frequent encounters with sequences of language make them more 'chunked' or 'fused' over time, which results in greater prominence of the chunk in relation to the individual components. However, it is imperative to point out that this does not entail that the parts within the whole are no longer available - they simply become less salient relative to the whole.

Such was the conclusion of Arnon and Cohen Priva (in press) whose study not only confirmed the effect of multi-word frequency in language production, but also contributed to our better understanding of the link between the parts and the whole and the prominence of the individual components within a larger chunk. Arnon and Cohen Priva (in press) investigated the effect of word and multi-word information on the duration of words in naturally elicited speech. Specifically, they explored whether or not the relationship between word and multi-word information changes across the frequency continuum. The authors hypothesised that if frequent three-word phrases are stored holistically, then the effect of component (i.e. individual word) frequency should disappear. On the contrary, if higher trigram frequency changes the prominence of individual word and multi-word information, then one should observe a reduced - but still significant - effect of individual word frequency and an increased effect of phrasal frequency for high frequency phrases. The authors observed a change in the prominence of word and multi-word information with growing phrase frequency. That is, for highly frequent trigrams, the effect of individual word frequency on phonetic duration decreased - but, critically, remained significant - while the effect of phrase frequency on phonetic duration increased (Arnon and Cohen Priva in press, Study 3). The authors concluded that repeated usage leads to a growing prominence of multi-word information - the finding that supports much of what is known about formulaic language; but that repeated usage does not eliminate the effect of word information - a novel finding, and the strongest evidence yet, that argues against the holistic storage for frequent phrases.

More evidence against holistic storage comes from another source - idiom priming. Psycholinguistic studies suggest that regular decompositional analyses are involved in the processing of idiomatic expressions. For example, using a syntactic priming paradigm, Konopka and Bock (2009) examined the effectiveness of idiomatic and non-idiomatic phrasal verbs (e.g. pull off a robbery, pull off a sweatshirt) in inducing structural generalisations. In three experiments, participants were asked to recall sentences they had read using rapid serial visual presentation. Prime and target sentences comprised phrasal verbs where the particle followed the verb (e.g. pull off a sweatshirt), or the direct object (e.g. pull a sweatshirt off). The idiomatic verbs used as primes were non-decomposable 
idioms; that is, the meaning of a verb cannot be inferred on the basis of the literal meaning of its components (e.g. pull off a robbery). Konopka and Bock (2009) found that idiomatic expressions undergo the same syntactic analysis and are as amendable to priming as literal phrases - a finding that offered further support to earlier studies (e.g. Cutting and Bock 1997; Peterson et al. 2001). These researchers concluded that if idioms call on generalised syntax (as was shown in the study), then other types of expressions (literal and compositional, more and less frequent) also rely on generalised syntax.

In a more recent study, Snider and Arnon (2012) had participants perform a sentence completion task in order to assess how idiomatic and non-idiomatic datives prime their syntactic construction during language production. Prime sentences contained the information that made participants complete the sentence as required (e.g. Idiomatic: The lifeguard gave the surfer [the creeps]/The lifeguard gave the creeps to [the surfer]; Non-idiomatic: The mother gave the hungry baby [some food]/The mother gave some food to [the hungry baby]). The target sentences contained a subject noun-phrase and a dative verb (e.g. The flight attendant gave) and could be completed with either the double object or the prepositional object. It was found that idiomatic phrases primed their syntactic construction just as non-idiomatic, compositional phrases did, implying that idioms (and, arguably, other types of formulaic language) possess internal structure (Experiment 2). The results of Snider and Arnon (2012) are similar to those of Konopka and Bock (2009), in that both studies established that idioms have internal structure and are governed by regular syntactic processes, comparable to non-idioms - an account that leaves little of the idea that idioms are stored and processed as holistic units (Snider and Arnon 2012).

In addition, regular decompositional analyses have been found to be involved in the processing of idiomatic expressions not only at the level of syntax (Konopka and Bock 2009; Snider and Arnon 2012), but also at the level of phonology and semantics (Sprenger et al. 2006). In a series of experiments, Sprenger and colleagues (2006) investigated lexical access during the production of idiomatic expressions (e.g. hit the road). In Experiment 1, participants produced idioms and control (literal) phrases in response to a visual prompt and an auditory prime, which was either identical to the target, or semantically and phonologically unrelated. It was found that both idioms and non-idioms can be primed successfully by priming one of the constituent words, and that during the planning of an idiom its constituents are accessed separately. In the subsequent experiments, Sprenger et al. (2006) found evidence for their hypothesis that literal word meanings become active during the production of idioms. Taken together, Sprenger et al.'s (2006) findings support the compositional nature of idioms. In that, they add to the body of research that has long rejected 
the idea that idiomatic expressions are processed in a holistic manner (e.g. Cacciari and Tabossi 1988; Gibbs et al. 1989; Titone and Connine 1999). As Gibbs and Nayak (1989) note, approaches which consider idioms to be "separate lexical entries fail to account for the productivity of certain idioms" (cited in Durrant 2008: 70).

Wray (2008) acknowledges that the findings of the idiom processing studies, such as those discussed above, argue against the view of holistic storage and processing. In response to these findings, she proposed a model of idiom processing that "offers a solution to the conundrum of how idioms are processed" (Wray 2008: 30). In this model, the idiom experimental findings are explained "as artificial engagements with the form" (Wray 2008: 32). That is, Wray (2008) argues, if we ask participants to look at or listen to idioms out of a communicative context, we should not find it surprising that they attend to idioms in a different way to how they would attend to them in a more naturalistic setting (e.g. during a conversation or when reading a book). However, it is noteworthy that most psycholinguistic experiments are designed such that participants are not given any information about the design or aims of the experiment (at least, not prior to the experiment); participants are, thus, blind to experimental manipulations. In addition, it is customary to have a large number of distractors (fillers) to ensure participants do not notice the presence of the experimental stimuli. Wray (2002) is, too, sceptical about experimental and clinical procedures, arguing that they may encourage participants' analytical strategies and are, thus, likely to fail to measure the effects of holistic processing. While this can certainly be true, we believe that a sound experimental design and a sensitive methodology (e.g. eyetracking and ERPs, ${ }^{6}$ see Roberts and Siyanova-Chanturia 2013; SiyanovaChanturia 2013) can help overcome many of the problems associated with testing in a laboratory setting. It is further worth noting that the questions pertaining to the mental lexicon, the processing of formulaic language, its holistic storage and retrieval - or lack thereof - can only ever be addressed by means of carefully conducted experimental manipulations. After all, how else can we tap into on-line language processing?

Finally, it is not just idioms that have been shown to be processed compositionally, but also other, less idiosyncratic and salient, types of formulaic language. Molinaro et al. (2013b) used event-related brain potentials (ERPs) to

6 Electroencephalography (EEG) is the recording of electrical activity produced by neurons in the brain. Event-related brain potentials (ERPs) are EEG responses time-locked to a particular stimulus and averaged over a large number of trials. ERPs plotted against post-stimulus time are represented by a series of positive and negative peaks (Van Petten and Kutas 1991). Such positive and negative waves are associated with different ERP components, where "a component is a reflection of the neural mechanisms involved in certain functional (i.e. cognitive or 
investigate the processing of complex prepositions (e.g. in the hands of) versus modified complex prepositions (e.g. in the capable hands of). Brain response was measured on the noun (hands) when it was part of the frequent unmodified and novel modified phrase. Molinaro and colleagues (2013b) observed a smaller $\mathrm{N} 400$ on the noun in the modified conditions (e.g. in the capable hands of), which was taken to suggest that the modification did not disrupt comprehension of the complex prepositions. It was argued that complex prepositions could be internally modified without losing their functional role. This finding is important because it indicates that individual words within a formulaic sequence can, and do, maintain their semantic and syntactic properties, despite being part of a highly conventional phrase. In consonance with Konopka and Bock (2009), Molinaro et al. (2013b) concluded that regular decompositional analyses were involved in the processing of complex prepositions such as in the hands of and in contrast with.

In another ERP study, Tremblay and Baayen (2010) investigated the processing of lexical bundles (e.g. in the middle of) whose frequencies ranged from very infrequent ( 0.03 occurrences per million words in the British National Corpus) to very frequent (105 occurrences per million words in the British National Corpus). Tremblay and Baayen (2010) found that the probability of occurrence of the fourword lexical bundles, and individual word and trigram frequency, significantly affected participants' response. Specifically, a phrasal frequency effect was found around 110-150 ms following stimulus presentation. This finding was linked to the P1 and N1 components, the ERP components normally associated with general attentional processes. Based on this finding, the authors concluded that frequent bundles "are retrieved in a holistic manner" (p. 170), and that more and less frequent four-word sequences are best viewed as two extremes on a frequency/probability continuum. While other researchers have also proposed that higher and lower frequency linguistic structures fall along the same frequency continuum and, thus, frequency should be viewed as a continuous rather than dichotomous (i.e. high vs. low) variable, it is questionable whether or not the modulations of the P100 and N100 amplitudes support the conclusion that frequent lexical bundles are "retrieved in a holistic manner" (p. 170), or, are suggestive of more general attentional processes. The authors' conclusion is particularly debatable given Molinaro et al.'s (2013b) observation that individual words within formulaic sequences maintain their properties, despite being part of a highly conventional phrase, a finding that corroborates the behavioural evidence (Konopka and Bock 2009; Snider and Arnon 2012; Sprenger et al.

perceptual) processes" (Kaan 2007: 573). For an overview of the studies using the ERP technique in the context of formulaic language, see Siyanova-Chanturia (2013). 
2006). In addition, it is unclear why Tremblay and Baayen (2010) chose not to focus on the N400 and the P300 components - the ERP components known to be implicated in frequency and predictability modulations in the context of phrasal processing (Vespignani et al. 2010; for a review, see SiyanovaChanturia 2013). Despite these shortcomings, however, the data presented in Tremblay and Baayen (2010) are important in that they suggest that highly frequent and predictable bundles can be accessed early on during on-line processing - as early as their initial parts - a finding that further argues for the central role of frequency and predictability in natural language processing (also, see Molinaro et al. 2013a, who found that highly constraining formulaic contexts elicit very early - as early as $120 \mathrm{~ms}$ post-stimulus - brain reactions compared to more compositional contexts).

In conclusion, while it has been demonstrated that due to their frequency and predictability formulaic sequences are processed quantitatively faster than matched control phrases (with reference to holistic storage: Jiang and Nekrasova 2007; Kim and Kim 2012; Millar 2011; Tremblay et al. 2011; Underwood et al. 2004; without reference to holistic storage: Arnon and Cohen Priva 2013; Arnon and Snider 2010; Siyanova-Chanturia et al. 2011a, Siyanova-Chanturia et al. 2011b), it is important to bear in mind that these studies were not designed to test the idea of chunks being processed as unanalysed wholes. So, caution is needed when interpreting their results and extending them beyond the speed of processing. Similar concerns have been voiced by other researchers. Arnon and Snider (2010) note that finding phrase-frequency effects should not be taken to suggest that various instances of formulaic language are stored and processed as unanalysed wholes. Likewise, Edmonds (2014) argues that the trend that faster processing is often equated with holistic storage is 'problematic'. She is critical of Underwood et al.'s (2004) and Jiang and Nekrasova's (2007) 'unequivocal' interpretations of their findings, noting that "there is not an exclusive relationship between facilitation and holistic storage" (Edmonds 2014: 91).

Critically, the majority of the studies whose findings can be used as an argument in the holistic storage debate (e.g. Arnon and Cohen Priva in press; Cutting and Bock 1997; Konopka and Bock 2009; Molinaro et al. 2013b; Peterson et al. 2001; Snider and Arnon 2012; Sprenger et al. 2006) have demonstrated that: (1) individual words within a formulaic sequence maintain their semantic and syntactic properties despite being part of a frequent configuration, (2) regular decompositional analyses are involved in the processing of formulaic sequences (both idioms and non-idioms), and (3) repeated usage of highly frequent phrases does not eliminate the effect of constituent word frequency. Clearly, this evidence argues against the proposition that formulaic sequences are stored as unanalysed wholes, or that they behave akin to a single 
morpheme. It is also clear, however, that more empirical evidence is needed if one wants to address the question of holistic storage and processing, for example, through the experimental paradigms that test activation, prominence, or modifiability of the individual components within a larger unit (as in Arnon and Cohen Priva in press; Konopka and Bock 2009; Sprenger et al. 2006), rather than focus solely on the speed of phrasal processing. As more empirical evidence emerges, it becomes ever more evident that equating faster processing times with holistic storage and processing is problematic, in that this view fails to account for what is known in psycholinguistic and neurolinguistic literature about the on-line processing of compounds, idioms, complex prepositions, collocations, and other kinds of formulaic language.

Last but not least, one may ask: Why does it matter if formulaic sequences were or were not stored as wholes? Would this knowledge change anything in our view of the role that phrasal frequency plays in language processing? As Arnon and Cohen Priva (in press) put it, the intuition that there is something special about formulaic sequences "can be captured without assuming that they are stored as one holistic unit, simply by allowing the whole to become more prominent with increased usage". There may simply be no need to argue for the holistic storage of recurrent sequences. After all, what matters most is the fact that the human processer is highly sensitive to frequency and probability distributions not only at the word but also at the phrase level, and that repeated phrase usage leads to a growing prominence of the whole relative to the parts - the findings that have far-reaching implications for how we notice, learn, process, and use language.

Acknowledgments: The author would like to thank the two anonymous Reviewers, as well as Phil Durrant, for their very helpful and insightful comments on the earlier version of this paper.

\section{References}

Altenberg, B.. 1998. On the phraseology of spoken English: The evidence of recurrent word combinations. In A. P. Cowie (ed.), Phraseology: Theory, analysis and applications, 101-122. Oxford: Oxford University Press.

Arnon, I. \& U. Cohen Priva. 2013. More than words: The effect of multi-word frequency and constituency on phonetic duration. Special Issue: Parsimony and Redundancy in Usage-Based Models of Linguistic Knowledge. Language and Speech 56 (3). 257-264.

Arnon, I. \& U. Cohen Priva. 2014. Time and again: The changing effect of word and multiword frequency on phonetic duration for highly frequent sequences. In Wulff, Stefanie and Debra Titone (eds.), Bridging the Methodological Divide: Linguistic and psycholinguistic approaches to formulaic language. Special issue of The Mental Lexicon 9 (3). 377-400. DOI: $10.1075 / \mathrm{ml}$.9.3.01arn 
Arnon, I. \& N. Snider. 2010. More than words: Frequency effects for multi-word phrases. Journal of Memory and Language 62. 67-82.

Badecker, W. 2001. Lexical composition and the production of compounds: Evidence from errors in naming. Language and Cognitive Processes 16. 337-366.

Bannard, C. \& D. Matthews. 2008. Stored word sequences in language learning: The effect of familiarity on children's repetition of four-word combinations. Psychological Science 19. 241-248.

Biber, D., S. Johansson, G. Leech, S. Conrad \& E. Finegan. 1999. Longman grammar of spoken and written English. Harlow: Longman.

Bod, R. 1998. Beyond grammar: An experience-based theory of language. Stanford, CA: Center for the Study of Language and Information.

Bod, R. 2006. Exemplar-based syntax: How to get productivity from exemplars. The Linguistic Review 23. 291-320.

Bybee, J. 1998. The emergent lexicon. Chicago Linguistic Society 34. 421-435.

Bybee, J. 2006. From usage to grammar: The mind's response to repetition. Language 82 (4). 711-733.

Cacciari, C. \& P. Tabossi. 1988. The comprehension of idioms. Journal of Memory and Language 27. 668-683.

Cutting, J. \& K. Bock. 1997. That's the way the cookie bounces: Syntactic and semantic components of experimentally elicited idiom blends. Memory and Cognition 25 (1). 57-71.

Dahlmann, I. \& S. Adolphs. 2007. Pauses as an indicator of psycholinguistically valid multiword expressions (MWEs)? Proceedings of the Workshop on a Broader Perspective of Multiword Expressions (pp. 49-56). Prague, Association for Computational Linguistics.

Durrant, P. 2008. High frequency collocations and second language learning. Nottingham: University of Nottingham PhD dissertation.

Durrant, P. \& A. Siyanova-Chanturia. in press. Learner corpora and psycholinguistic research. In S. Granger, G. Gilquin, \& F. Meunier (eds.), Cambridge handbook of learner corpus research. Cambridge: Cambridge University Press.

Edmonds, A. 2014. Conventional expressions. Investigating pragmatics and processing. Studies in Second Language Acquisition 36 (1). 69-99.

Erman, B. \& B. Warren. 2000. The idiom principle and the open choice principle. Text 20 (1). $29-62$.

Gibbs, R. \& N. Nayak. 1989. Psycholinguistic studies on the syntactic behaviour of idioms. Cognitive Psychology 21. 100-138.

Gibbs, R., N. Nayak \& C. Cutting. 1989. How to kick the bucket and not decompose: Analyzability and idiom processing. Journal of Memory and Language 28. 576-593.

Goldberg, A. 1995. Constructions. Chicago: University of Chicago Press.

Goldberg, A. 2006. Constructions at work: The nature of generalization in language. Oxford: Oxford University Press.

Herbst, T. 2011. Choosing sandy beaches - collocations, probabemes and the idiom principle. In T. Herbst, S. Faulhaber, \& P. Uhrig (eds.), The phraseological view of language. A tribute to John Sinclair, 27-57. Berlin: De Gruyter Mouton.

Hoey, M. 2005. Lexical priming: A new theory of words and language. Oxford: Routledge.

Jiang, N. \& T. M. Nekrasova. 2007. The processing of formulaic sequences by second language speakers. The Modern Language Journal 91 (3). 433-445. 
Juhasz, B. 2007. The influence of semantic transparency on eye movements during English compound word recognition. In R. van Gompel, M. Fischer, W. Murray, \& R. Hill (eds.), Eye movements: $A$ window on mind and brain, 374-389. Oxford: Elsevier Science.

Kaan, E. 2007. Event-related potentials and language processing: A brief overview. Language and Linguistics Compass 1 (6). 571-591.

Kapatsinski, V. \& J. Radicke. 2009. Frequency and the emergence of prefabs: Evidence from monitoring. In R. Corrigan, E. Moravcsik, H. Ouali, \& K. Wheatley (eds.), Formulaic language, 499-522. Amsterdam, the Netherlands: John Benjamins.

Kim, S. H. \& J. H. Kim. 2012. Frequency effects in L2 multi-word unit processing: Evidence from self-paced reading. TESOL Quarterly 46 (4). 831-841.

Konopka, A. \& K. Bock. 2009. Lexical or syntactic control of sentence formulation? Structural generalizations from idiom production. Cognitive Psychology 58 (1). 68-101.

Kuperman, V., R. Bertram \& R. H. Baayen. 2008. Morphological dynamics in compound processing. Language and Cognitive Processes 23. 1089-1132.

Kuperman, V., R. Schreuder, R. Bertram \& R. H. Baayen. 2009. Reading polymorphemic Dutch compounds: Toward a multiple route model of lexical processing. Journal of Experimental Psychology 35. 876-895.

Langacker, R. 1987. Foundations of cognitive grammar, Vol. 1. Stanford: Stanford University Press.

Libben, G. 1998. Semantic transparency in the processing of compounds: Consequences for representation, processing, and impairment. Brain and Language 61. 30-44.

Millar, N. 2011. The processing of malformed formulaic language. Applied Linguistics 32 (2). 129-148.

Molinaro, N., P. Barraza \& M. Carreiras. 2013a. Long-range neural synchronization supports fast and efficient reading: EEG correlates of processing expected words in sentences. Neurolmage 72. 120-132.

Molinaro, N., P. Canal, F. Vespignani, F. Pesciarelli \& C. Cacciari. 2013b. Are complex function words processed as semantically empty strings? A reading time and ERP study of collocational complex prepositions. Language and Cognitive Processes 28 (6). 762-788.

Molinaro, N. \& M. Carreiras. 2010. Electrophysiological evidence of interaction between contextual expectation and semantic integration during the processing of collocations. Biological Psychology 83 (3). 176-190.

Mondini, S., C. Luzzatti, P. Saletta, N. Allamano \& C. Semenza. 2005. Mental representation of prepositional compounds: Evidence from Italian agrammatical patients. Brain and Language 94. 178-187.

Pawley, A. \& F. H. Syder. 1983. Two puzzles for linguistic theory: Nativelike selection and Nativelike fluency. In Richards, J. C. \& Schmidt, R. W. (eds.), Language and communication, 191-226. New York: Longman.

Peterson, R., C. Burgess, G. Dell \& K. Eberhard. 2001. Disassociation between syntactic and semantic processing during idiom comprehension. Journal of Experimental Psychology: Learning, Memory, and Cognition 27 (5). 1223-1237.

Pierrehumbert, J. 2001. Exemplar dynamics: Word frequency, lenition and contrast. In J. Bybee \& P. Hopper (eds.), Frequency and the emergence of linguistic structure, 137-157. Amsterdam, the Netherlands: Benjamins.

Pollatsek, A., J. Hyönä \& R. Bertram. 2000. The role of morphological constituents in reading Finnish compound words. Journal of Experimental Psychology: Human Perception and Performance 26. 820-833. 
Raupach, M. 1984. Formulae in second language speech production. In H. W. Dechert, D. Mole, \& M. Raupach (eds.), Second language productions, 114-137. Tubingen, Germany: Gunter Narr Verlag.

Roberts, L. \& A. Siyanova-Chanturia. 2013. Using eye-tracking to investigate topics in L2 acquisition and L2 sentence and discourse processing. [Special issue]. Studies in Second Language Acquisition 35 (2). 213-235.

Schmitt, N. \& R. Carter. 2004. Formulaic sequences in action: An introduction.

In N. Schmitt (ed.), Formulaic sequences: Acquisition, processing, and use, 1-22.

Philadelphia, PA: John Benjamins.

Siyanova-Chanturia, A. 2013. Eye-tracking and ERPs in multi-word expression research:

A state-of-the-art review of the method and findings. The Mental Lexicon

8 (2). $245-268$.

Siyanova-Chanturia, A., K. Conklin \& N. Schmitt. 2011a. Adding more fuel to the fire: An eyetracking study of idiom processing by native and nonnative speakers. Second Language Research 27. 251-272.

Siyanova-Chanturia, A., K. Conklin \& W. van Heuven. 2011b. Seeing a phrase 'time and again' matters: The role of phrasal frequency in the processing of multi-word sequences. Journal of Experimental Psychology: Language, Memory and Cognition 37. 776-784.

Siyanova-Chanturia, A. \& R. Martinez. 2014. The idiom principle revisited. Applied Linguistics. doi: $10.1093 /$ applin/amt054.

Siyanova-Chanturia, A. \& S. Spina. in press. Investigation of native speaker and second language learner intuition of collocation frequency. Language Learning.

Snider, N. \& I. Arnon. 2012. A unified lexicon and grammar? Compositional and non-compositional phrases in the lexicon. In S. Gries \& D. Divjak (eds.), Frequency effects in language, 127-163. Berlin: Mouton de Gruyter.

Sosa, A. \& J. MacFarlane. 2002. Evidence for frequency-based constituents in the mental lexicon: Collocations involving the word of. Brain and Language 83. 227-236.

Sprenger, S., W. Levelt \& G. Kempen. 2006. Lexical access during the production of idiomatic phrases. Journal of Memory and Language 54. 161-184.

Spöttl, C. \& M. McCarthy. 2004. Comparing knowledge of formulaic sequences across L1, L2, L3, and L4. In N. Schmitt (ed.), Formulaic sequences: Acquisition, processing, and use, 191-225. Philadelphia, PA: John Benjamins.

Titone, D. \& C. Connine. 1999. One the compositional and noncompositional nature of idiomatic expressions. Journal of Pragmatics 31. 1655-1674.

Tomasello, M. 2003. Constructing a language: A usage-based theory of language acquisition. Cambridge, MA \& London: Harvard University Press.

Tremblay, A. \& R. H. Baayen. 2010. Holistic processing of regular four-word sequences: A behavioral and ERP study of the effects of structure, frequency, and probability on immediate free recall. In D. Wood (ed.), Perspectives on formulaic language: Acquisition and communication, 151-173. London: The Continuum International Publishing Group.

Tremblay, A., B. Derwing, G. Libben \& C. Westbury. 2011. Processing advantages of lexical bundles: Evidence from self-paced reading and sentence recall tasks. Language Learning 61 (2). 569-613.

Underwood, G., N. Schmitt \& A. Galpin. 2004. The eyes have it: An eye-movement study into the processing of formulaic sequences. In N. Schmitt (ed.), Formulaic sequences, 153-172. Amsterdam, the Netherlands: John Benjamins. 
Van Petten, C. \& M. Kutas. 1991. Influences of semantic and syntactic context in open-and closed-class words. Memory and Cognition 19. 95-112.

Vespignani, F., P. Canal, N. Molinaro, S. Fonda \& C. Cacciari. 2010. Predictive mechanisms in idiom comprehension. Journal of Cognitive Neuroscience 22 (8). 1682-1700.

Wray, A. 2002. Formulaic language and the lexicon. Cambridge: Cambridge University Press.

Wray, A. 2008. Formulaic language: Pushing the boundaries. Oxford: Oxford University Press.

\section{Bionote}

\section{Anna Siyanova-Chanturia}

Anna Siyanova-Chanturia is a Lecturer in Linguistics in the School of Linguistics and Applied Language Studies, Victoria University of Wellington, New Zealand. Anna works in the fields of second language acquisition, corpus linguistics, and psycholinguistics. Anna's primary research interests include cognitive and psychological aspects of second language acquisition, learner corpus research, bilingualism, usage-based theories, phraseology, and vocabulary. In particular, Anna's research investigates acquisition, processing, and use of multi-word expressions (e.g., idioms, collocations, binomials, lexical bundles, multi-word verbs, etc.) in a first and second language. 